\title{
Bare footprint analysis comparing two collection methods adopting the reel measurement system and adobe photoshop
}

\begin{abstract}
This study examined the role that dissimilar bare footprint collection and measurement processes have on the Reel method of measurement in forensic podiatry and its use in the Criminal Justice System. Previous research indicated the Reel method was a valid and reliable measurement system for bare footprint analysis but various collection systems had been used to collect footprint data and both manual and digital measurement processes were utilized in forensic podiatry and other disciplines. This study contributes to the debate about collecting bare footprints; the techniques employed to quantify various Reel measurements and considered whether there was asymmetry between footprints of the same person. An inductive, quantitative paradigm used two gathering procedures for footprint collection and the subsequent dynamic footprints subjected to Adobe $(\mathrm{C}$ Photoshop(C measurement of a truncated version of the Reel variables. The findings from the research suggested there were no statically significant differences in the footprint measurements using dissimilar collection devices, that Adobe (C) Photoshop $(\mathcal{C}$ technique for measurement offers a reasonably easy method for measurements and offers alternative findings to that of Reel using a comparative digital measuring arrangement. Standard Error of Mean showed variation between feet/footprints and between collection systems and the findings provide support for the Reel study.
\end{abstract}

Keywords: bare footprint, Adobe $($ photoshop $\subset$, comparison, analysis, reel measurement, forensic podiatry
Volume 2 Issue 2 - 2016

\author{
J Gordon Burrow \\ Department of Health and Social Care, Glasgow Caledonian \\ University, UK
}

Correspondence: J Gordon Burrow, Department of Health and Social Care, Glasgow Caledonian University, 4 Borebrae, Newmilns, Ayrshire, Scotland, UK, Tel 44 I560322753, Email gordon.burrow@btinternet.com

Received: February 08, 2016 | Published: February 26, 2016
Abbreviations: SEM, standard error of mean; CJS, criminal justice system; Std Dev, standard deviation; CCTV, closed circuit television

\section{Introduction}

The human foot and its impressions have been used by anthropologists and others to forensically determine identification by amongst other measures, height, ${ }^{1-6}$ weight $^{7-13}$ or gender ${ }^{14-17}$ of a person. However, concerns about the rigour of the scientific methods and testimony of forensic experts in a number of forensic fields have been raised in some Courts. ${ }^{18,19}$ Naples et al., ${ }^{20}$ suggest, however, that: "Forensic science provides evidence to resolve legal issues through the application of scientific principles". Forensic science is normally conducted by forensic scientists, whose primary objective is the evenhanded or non-biased use of all available information to determine the facts and ultimately the truth no matter who they are instructed by ${ }^{21}$ Forensic scientists and forensic science analysis is concerned with the conclusions that can be logically deduced from the evidence, as well as those that cannot. Thus, consideration is afforded to the strengths as well as limitations of the physical evidence. ${ }^{21}$ According to Morrison, ${ }^{22}$ forensic science is in the midst of a paradigm shift "in the evaluation and presentation of evidence in the forensic sciences which deal with the comparison of the quantifiable properties of objects of known and questioned origin.... highlighting the transformation involved in moving from a pre-science to an empirically grounded science". This new paradigm echoes the requirements for admissibility of scientific evidence set out in the US Supreme Court ruling in Daubert v Merrell Dow Pharmaceuticals (92-102) although the Daubert principle is not universally accepted within the United Kingdom.
It is also to some extent based on the 2009 National Research Council report in the USA,,$^{23}$ which was derogatory of the 'scientific' basis for a number of eminent forensic sciences. However, the "new thinking' meets those requirements of the National Occupational Standards Skills for Justice suite ${ }^{24}$ and the Forensic Science Regulator in the United Kingdom. ${ }^{25}$ Forensic podiatry uses the skills and attributes of the podiatric profession and applies these attributes and specialized knowledge to the development of evidence through scientific and technical investigation to assist courts in resolving questions of fact in civil or criminal trials. According to Vernon et al., ${ }^{26}$ forensic podiatry is: "the application of sound and researched podiatry knowledge and experience in forensic investigations, to show the association of an individual with a scene of crime, or to answer any other legal question concerned with the foot or footwear that requires knowledge of the functioning foot". Practically, forensic podiatrists help with identification or elimination of suspects of crime from footprints and footwear. This is particularly true where foot function and wear marks are linked with the foot/shoe boundary and shoe size disputes may be involved. They may also utilize specialist knowledge of the lower limb function to draw a parallel with the gait patterns of individuals captured on Closed Circuit Television (CCTV) (unknown footage) with the gait patterns of suspected offenders (known footage).

The forensic podiatrist must ensure all examinations are complete, the tests performed correctly, the analysis of the scientific data is thorough and the written report is correct and easily understood by the layperson. Finally, they must ensure the evidence given is complete, unbiased and truthful; anything less being unacceptable. Again, this need for unbiased scientific objectivity is reiterated by Dror ${ }^{27}$ who 
encourages forensic scientists to develop strict protocols and detailed scientific methods, thus forensic podiatry is at the forefront of that paradigm shift. 'Footprints' are the impressions/marks/images left behind by a person walking, whereas 'shoeprint' is the specific term for prints made by shoes. This article focuses on the role of the bare footprint in crime scene identification and will be determining the latent two-dimensional impression evidence, rather than three-dimensional casted evidence. Forensic podiatry is a relatively new forensic science discipline ${ }^{28}$ starting in the 1970's in Canada and the United Kingdom. Podiatrists were employed to determine if identification of suspects was possible from foot impressions left at a crime scene.

The role and scope of forensic podiatry has now been clarified and agreed by the International Association of Identification ${ }^{29}$ whilst the standards for forensic podiatry are currently being developed under the auspices of the Special Advisory Group of the College of Podiatry in the United Kingdom and associated with the Forensic Science Regulator. This study used the exploratory and empirical research methodologies for its epistemological assumption, which was that the foot and its dimensions are open to measurement using anthropometry or valid and reliable anthropometric measurements. However, the ruling of Courts on the admissibility of evidence is based on a variety of decisions. These may be viewed as competing, but may be complementary and allow the development of specialisms within the forensic science community, which do not currently have a basis in the hard science but that utilize the scientific principles of theory testing and experimentation. As an expert witness in criminal proceedings, forensic podiatrists must be aware of the discipline's methodological variations and limitations, especially in light of the 2009 NRC Report in the USA ${ }^{23}$ and the Law Commissions Report in the United Kingdom. ${ }^{30}$

In their report the Law Commission reported that: "We explained that the common law approach to the admissibility of expert opinion evidence is one of laissez-faire, with such evidence being admitted without sufficient regard to whether or not it is sufficiently reliable to be considered by a jury. We concluded that this is unsatisfactory and proposed that the common law approach should be replaced by a new admissibility test set out in primary legislation". Currently in England and Wales, expert evidence testimony can be applied if the first three limbs of the common law test were applied. The witness giving expert evidence of fact or opinion should be able to do so, only if the court is likely to need such assistance, the witness is an expert in the relevant field and the witness will provide impartial, objective evidence (that is, a sufficiently complete account as opposed to a partial picture distorted by bias)..$^{30}$

The fourth limb of the admissibility of evidence (evidentiary reliability) was the principal issue in the Law Commission consultation paper. That principle is concerned with the reliability of opinion evidence provided by an impartial, properly qualified expert. Unknown or questioned footmarks/footprints, from crime scenes, are directly compared with footprints of a known individual (or suspect) and a conclusion reached as to whether these marks share common ownership. Plantar prints or bare foot impressions are essential to this process, although where a footwear item is involved, marks from the borders of the forefoot and the dorsum of the toes may also be considered and analyzed. ${ }^{28}$ According to DiMaggio ${ }^{31}$ the basis of forensic investigation is that of evaluation, leading to a determination (or not) of a common origin between two specimens, which may establish positive identification. In this study the following goals were set: i. To collect a set of footprints under controlled conditions from a group of young adults.

ii. To perform measurements, digitally using Adobe $(\mathcal{C}$ Photoshop $\mathbb{C}$, on the footprints following a rigorous protocol.

iii. To examine differences between footprint measurements derived from different collection systems.

iv. To meet the goals a number of objectives were set:

v. To select and carefully define a footprint measurement technique.

vi. To record footprints from a group of young adults using the defined collection techniques.

vii. To establish which of the collection methods implemented was the most reliable for the assessment of footprint measurements.

\section{Materials and methods}

\section{Design}

The study design or methodology was that of a quantitative test re-test design, describing a method whereby linear measurements were used to determine various lengths of anatomical structures and regions of the foot and compared with other measurements for other footprints to determine if there was any correlation or similarity between different sample footprints.

\section{Ethics}

Ethical approval for the study was obtained from the School of Health, Glasgow Caledonian University ethics Committee and an appropriate Risk Assessment was conducted using a simple pro-forma to ensure that the risk of injury to anyone taking part was minimized in line with Research Governance procedures.

\section{Sample}

Desirable subjects for the study were defined as anyone who did not have medical or surgical problems that would affect their gait or walking and that left and right footprints could be obtained from them with normal morphological features of the human foot anticipated. It was decided that the population would be that of young people between the ages of 18 and 65 residing in the United Kingdom and regarding themselves as British. The sampling frame however was that of University students/staff as the researcher had access to that sampling frame. Non-probability self-selection sampling utilized the subjective judgment of the researcher and was a valuable sampling technique. As a sampling strategy, self-selection sampling can be used within a wide range of research designs and research methods. ${ }^{32}$ The technique was applicable for this study in that the interest of the study was to determine if there were differences between collection methods and digital processes of measurement rather than making generalizations or statistical inference to the wider population of feet. The research was exploratory in nature, therefore the need for probability sampling was not deemed necessary. The key component was that research subjects volunteered to take part in the research rather than being approached by the researcher directly.

\section{Selection criteria}

The following criteria were applied in selecting the study participants: 


\section{Inclusion criteria}

i. Must have two feet.

ii. Participants were between 16 and 65 years of age.

iii. Be of any gender or ethnic background.

iv. Be able to walk a minimum of 10 metres unsupported.

v. Have no medical or surgical condition that affected their walking pattern.

\section{vi. No current reported health issues}

\section{Exclusion criteria}

i. Any lower limb abnormality that would prevent 'normal' walking.

ii. Prosthetic $\operatorname{limb}(\mathrm{s})$.

iii. Persons who had difficulty standing steady or straight, and participants with hairstyle or head dress that prevented proper use of the height measuring equipment (a stadiometer).

\section{iv. Pregnancy.}

\section{Data collection}

Each participant was asked to provide informed consent to take part in the study. This included the researcher ensuring that the participant had no outstanding questions and that they were fully aware of the project. A unique identity was assigned to the participant and preliminary checks undertaken to confirm participant suitability.

Foot print collection and analysis: For this study two methods of acquiring dynamic footprints were assessed:

I. The Podotrack, a carbonized paper and cardboard base system

\section{The Inkless Shoe print system}

A $4^{\text {th }}$ step protocol was used in both cases of obtaining dynamic footsteps for both collection systems as the $4^{\text {th }}$ step falls between the step recommended by some authors ${ }^{33}$ and the step chosen by Reel in their reliability study. ${ }^{34,35}$ To compare the two techniques of acquiring the footprint a common method of measuring the footprints was used. The method used for measuring dimensions on the footprint was the Reel Method measurement protocol. ${ }^{35}$ The two different collection methods for footprints produce different footprints on initial observation (Figure 1) and an aim of the study was to determine which method of capture, Podotrack or Inkless allowed consistent, repeatable measurements using the Reel method of measurement.

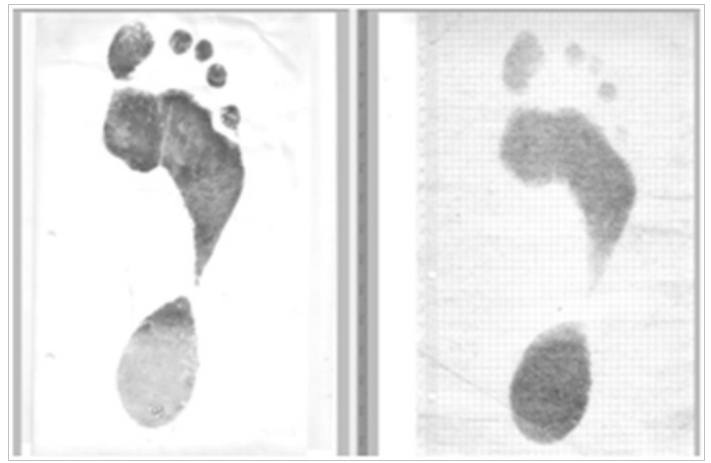

Figure I Two images of the same foot taken with the two different capturing systems-left hand side the Inkless system, right hand side Carbon paper system (Podotrack)
The Reel method uses a system of linear and angular measurements based on a validity and reliability study, which showed standard errors of means and other appropriate statistical analyses, which demonstrated the requirements for a valid, reliable method. ${ }^{35}$ Reel et al., ${ }^{35}$ initially started with defining the inner and outer tangents of the footprint, which were identified and bisected to create the central axis. A grid was placed over the image, which was then rotated to ensure the central axis was vertically aligned. A horizontal mark was then introduced which would cross the most proximal pixel of the heel in this new alignment This determined how the midpoint of the rear most aspect of the heel was defined and determined and the original lines of the Gunn method were also employed. ${ }^{36}$ From that point where the central axis and the heel intersect, a series of five lines were drawn to the apices of each toe (Figure 2).

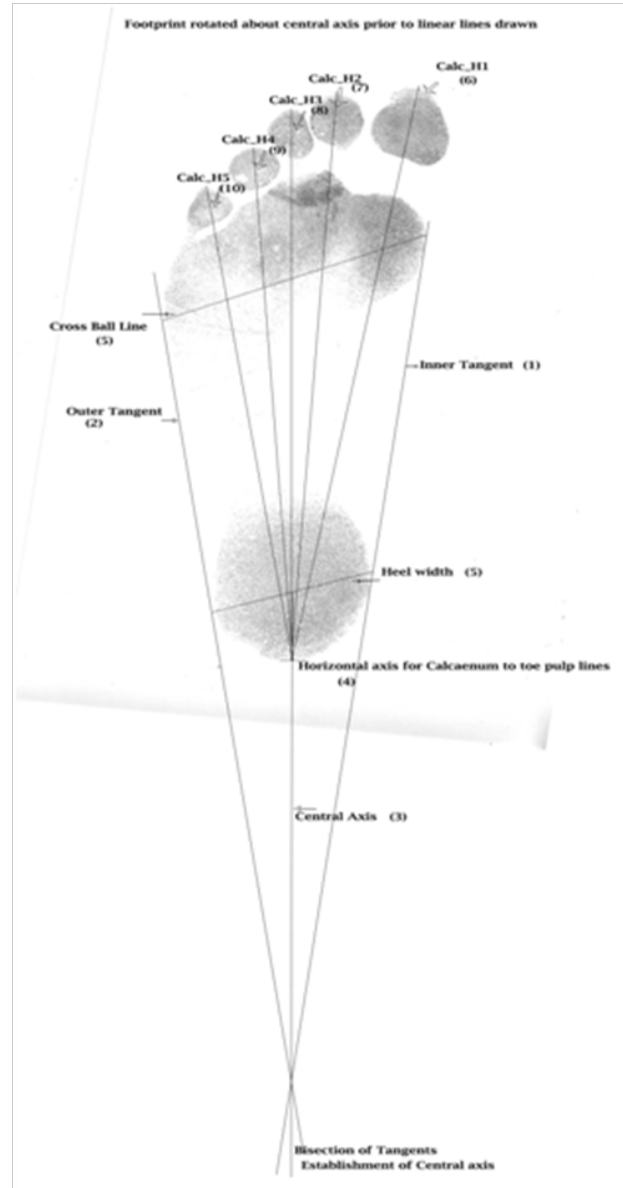

Figure 2 Reel lines for measurement showing inner and outer tangents and central axis, rotation of the footprint then establishing horizontal line for further lines.

Once the footprints were collected they were scanned so that they could be opened in Adobe (C) Photoshop $(\mathrm{CS} 5$ and CC for analysis. Images were then saved as TIF files. The TIF format was used as it allowed the original digitized image to be reconstructed exactly. Other formats (e.g., jpeg) do not allow this reconstruction as they use compression, which leads to loss of data and resolution in the image. Adobe $($ P Photoshop(C) Extended CS 5.1 and CC 2015 were used as the image analysis software of choice, as according to SWGIT [37] 'Software used in processing and analysing digital images should produce consistent results, permitting comparably trained personnel to achieve comparable analytical results'. Adobe $\subset$ Photoshop $\subset$ allowed 
for interpretation and examination of the scanned footprint image where interpretation, as used here, follows the Guidance of SWGIT, in that it "is the application of specific subject matter expertise to draw conclusions about subjects or objects depicted in images. Examples include a podiatrist drawing conclusions about foot shape from an image. ${ }^{38}$

\section{Data analysis}

Data measurements were saved as Excel files then transferred to IBM Statistical Package for Social Science (Version 22) for statistical analysis along with AnalystSoft Inc., StatPlus: mac- statistical analysis program for Mac OS. Version v5.

The study determined differences between collection methods and digital measurements (groups). The statistical tests were used to:

A. Determine whether there were differences between two or more groups of related and/or unrelated (independent) cases on a dependent variable; and

B. If such differences existed, determine where these differences lay.

Statistical tests were used to determine whether there were differences between groups and used within-subjects designs that involved related groups, e.g. the participants were always the same but the independent variables of footprint collection systems and methods of measurements were different and the dependent variables were the seven Reel measurements calculated.

Paired-sample t-tests with their subsequent assumptions were conducted. A paired-sample t-test assumes one dependent variable measured on the continuous level, in this case each of the seven Reel

Table I Demographics for the sample group measurements. The second assumption, one independent variable, consisted of two categorical or matched pairs-the test and re-test matched pairs. During the test there were no significant outliers and the distribution of the differences between dependent variables of the two related groups were approximately normally distributed. Pearson product-moment correlation was also used for comparing and assessing the association between measurements of the seven variables of the Podotrack with those of the Inkless shoe print collection system. The Pearson's correlation coefficient measures the strength and direction of the linear relationship between the variables and its values can range from -1 for a perfect negative linear relationship to +1 for a perfect positive linear relationship. The magnitude of the Pearson correlation coefficient determines the strength of the correlation. The guidelines provided by Cohen $^{39}$ were used in this study.

\section{Results and discussion}

The sample consisted of thirty-five individuals who all gave consent and data was collected from all of them. This was a selfselection sample of staff and students. The sample consisted of thirty-one females and four males. The age of the participants ranged from eighteen to fifty years of age with an average age of twentyfour point five years. The heights and weights of the sample showed a normal distribution curve using a Shapiro-Wilk's test for normality suggesting a normal distribution of height and weight for the sample. The results of the height can also be shown for the different genders, which showed slight dissimilarity in some basic descriptive statistical data. The mean of males was $172.0 \mathrm{~cm}$ (StdDev 7.5; SEM 3.8) compared with $161.3 \mathrm{~cm}$ for females (StdDev 6.2; SEM 1.1) (Table 1). For the whole sample weight was normally distributed (Shapiro Wilkes $\mathrm{p}>=0.05$ ) with males being on average heavier than females (Table 1).

\begin{tabular}{lllllll}
\hline & \multicolumn{2}{l}{ Weight* Kg } & \multicolumn{2}{l}{ Height* cm } & \multicolumn{2}{l}{ BMI* } \\
\cline { 2 - 7 } Male (4) Female (3I) & Male & Female & Male & Female & Male & Female \\
\hline Mean & 70 & 67.94 & 172 & 161.3 & 23.7 & 26.1 \\
Standard Deviation (StdDev) & 6.8 & 13.67 & 7.5 & 6.2 & 1.2 & 4.7 \\
Standard Error of Mean (SEM) & 3.4 & 2.45 & 3.8 & 1.1 & 0.6 & 0.8 \\
Minimum & 60.6 & 41.9 & 164 & 147 & 22.5 & 18.6 \\
Maximum & 75.7 & 107.8 & 182 & 178.5 & 25 & 37.4 \\
Shapiro-Wilks p value & 0.443 & 0.083 & 0.525 & 0.85 & 0.55 & 0.089 \\
\hline
\end{tabular}

Weight showed variations between genders with male weights being more closely grouped together and a more diverse weight range in females, identified by the minimum and maximum range, which can be seen to be greater for females than males, with one whose weight was above $100 \mathrm{~kg}$. In terms of BMI, females tended to be in the higher BMI category and at the higher end of the normal range. A Pearson's product moment correlation was run to assess any relationship between height, weight and BMI. Preliminary analysis showed the relationship to be linear with variables being normally distributed, as assessed by the Shapiro-Wilk test $(p>0.05)$ with no outliers. Height was moderately correlated with weight in the group, $r(35)=0.455, p<0.006$ while weight was strongly correlated with BMI, $\mathrm{r}(35)=0.875, \mathrm{p}<0.005$.

Footprints were collected from all the participants, from both feet, using two different collection processes and were then subjected to a digital method of measurement using the Reel system of measurement. The two collection processes were those of the Podotrack and the Inkless shoe print system. Each footprint of each foot was collected twice in a test re-test design and then each footprint was scanned and digitally measured using Adobe $(\subset$ Photoshop $($. There were 35 participants each having both feet collected twice using two systems: For the digital measurements, all dependent variables of the Reel measurements were recorded and the test and re test variables submitted to a paired-sample t-test, which was used to determine whether the mean difference between paired observations was statistically significantly different from zero. The right and left foot measurements were also compared. Differences in scores between the two tests (test and re-test) for both feet were conducted and used to conduct further tests to determine outliers and normality. Outliers were detected in a box plot. 


\section{Podotrack collection footprints}

Data was normally distributed for both feet data as assessed by visual inspection of a Normal Q-Q Plot. Data are mean \pm standard deviation, unless otherwise stated. Participants re-test tended to show a slight increase in size from the test scores in some variables whilst others inclined to demonstrate a decrease as demonstrated in (Table 2). The main predisposition for decrease in scores was for the right heel width and Calc_H3 of the left foot. All other measurements as demonstrated in Table 2 indicated a tendency for an increase between test and repeat test scores. However, outcomes of the Paired Sample test table demonstrated there were no statistically significant differences between test scores $(p>0.05)$ in all variables tested e.g., Calc_H4 left foot demonstrated $\mathrm{t}(34)=1.098, \mathrm{p}=0.280$ as shown in Table 3. The Standard Error of Mean (SEM) was between 1.36 and $.035 \mathrm{~cm}$, the right foot showed smaller SEMs than the left foot.

Table 2 Paired-sample statistics for the digital measurements between tests for the Podotrack footprints

\begin{tabular}{|c|c|c|c|c|c|}
\hline \multicolumn{2}{|c|}{ R-Right Foot; L- Left Foot } & \multirow{2}{*}{$\begin{array}{l}\text { Mean cm } \\
5.123\end{array}$} & \multirow{2}{*}{$\begin{array}{l}\mathbf{N} \\
35\end{array}$} & \multirow{2}{*}{$\begin{array}{l}\text { Std.Dev } \\
0.503\end{array}$} & \multirow{2}{*}{$\begin{array}{l}\text { Std. Error Mean } \\
0.085\end{array}$} \\
\hline R HW & Re test & & & & \\
\hline & Test & 6.424 & 35 & 7.605 & 1.285 \\
\hline \multirow[t]{2}{*}{$\mathrm{R} C B \mathrm{~L}$} & Re test & 9.105 & 35 & 0.598 & 0.101 \\
\hline & Test & 9.083 & 35 & 0.632 & 0.106 \\
\hline \multirow[t]{2}{*}{ R Calc_HI } & Re test & 24.546 & 35 & 1.356 & 0.229 \\
\hline & Test & 24.46 & 35 & 1.343 & 0.227 \\
\hline \multirow[t]{2}{*}{ R Calc_H2 } & Re test & 24.107 & 35 & 1.198 & 0.202 \\
\hline & Test & 24.051 & 35 & 1.29 & 0.218 \\
\hline \multirow[t]{2}{*}{ R Calc_H3 } & Re test & 22.52 & 35 & 4.104 & 0.693 \\
\hline & Test & 22.493 & 35 & 4.1 & 0.693 \\
\hline \multirow[t]{2}{*}{ R Calc_H4 } & Re test & 21.289 & 35 & 3.871 & 0.654 \\
\hline & Test & 21.26 & 35 & 3.869 & 0.654 \\
\hline \multirow[t]{2}{*}{ R Calc_H5 } & Re test & 19.696 & 35 & 3.577 & 0.604 \\
\hline & Test & 19.658 & 35 & 3.568 & 0.603 \\
\hline \multirow[t]{2}{*}{ L HW } & Re test & 6.528 & 35 & 8.061 & 1.361 \\
\hline & Test & 5.184 & 35 & 0.517 & 0.083 \\
\hline \multirow[t]{2}{*}{ L CBL } & Re test & 9.1 & 35 & 0.545 & 0.092 \\
\hline & Test & 9.077 & 35 & 0.563 & 0.095 \\
\hline \multirow[t]{2}{*}{ L Calc_HI } & Re test & 24.653 & 35 & 1.274 & 0.215 \\
\hline & Test & 24.591 & 35 & 1.311 & 0.221 \\
\hline \multirow[t]{2}{*}{ L Calc_H2 } & Re test & 24.265 & 35 & 1.165 & 0.196 \\
\hline & Test & 24.212 & 35 & 1.236 & 0.208 \\
\hline \multirow[t]{2}{*}{ L Calc_H3 } & Re test & 23.367 & 35 & 1.177 & 0.198 \\
\hline & Test & 22.67 & 35 & 4.131 & 0.698 \\
\hline \multirow[t]{2}{*}{ L Calc_H4 } & Re test & 21.483 & 35 & 3.893 & 0.658 \\
\hline & Test & 20.767 & 35 & 5.311 & 0.897 \\
\hline \multirow[t]{2}{*}{ L Calc_H5 } & Re test & 19.168 & 35 & 4.861 & 0.821 \\
\hline & Test & 18.525 & 35 & 5.814 & 0.982 \\
\hline
\end{tabular}

*Data normally distributed (Shapiro Wilkes $\mathrm{p}>=0.05$ )

Table 3 Paired Sample Test results for digital measurements of Podotracks footprints

\begin{tabular}{|c|c|c|c|c|c|c|c|}
\hline \multicolumn{2}{|c|}{ R-Right Foot L- Left Foot } & \multicolumn{3}{|c|}{ Paired Differences mm } & \multirow[t]{2}{*}{$\mathbf{t}$} & \multirow[t]{2}{*}{ df } & \multirow[t]{2}{*}{ Sig. (2-Tailed) } \\
\hline & & Mean & Std.Dev & Std. Error Mean & & & \\
\hline R HW & Re-test - test & -1.301 & 7.6 & 1.291 & -1.007 & 34 & 0.321 \\
\hline $\mathrm{R} C B \mathrm{~L}$ & Re-test - test & 0.022 & 0.21 & 0.035 & 0.63 & 34 & 0.533 \\
\hline
\end{tabular}




\begin{tabular}{|c|c|c|c|c|c|c|c|}
\hline \multicolumn{8}{|l|}{ ble conti } \\
\hline \multicolumn{2}{|c|}{ R-Right Foot L- Left Foot } & \multicolumn{3}{|c|}{ Paired Differences mm } & \multirow{2}{*}{$\begin{array}{l}\mathbf{t} \\
\mathrm{I} .248\end{array}$} & \multirow{2}{*}{$\begin{array}{l}\text { df } \\
34\end{array}$} & \multirow{2}{*}{$\begin{array}{l}\text { Sig. (2-Tailed) } \\
0.221\end{array}$} \\
\hline R Calc_HI & Re-test - test & 0.087 & $0.4 I$ & 0.069 & & & \\
\hline R Calc_H2 & Re-test - test & 0.056 & 0.46 & 0.071 & 0.782 & 34 & 0.44 \\
\hline R Calc_H3 & Re-test - test & 0.027 & 0.24 & 0.039 & 0.676 & 34 & 0.504 \\
\hline R Calc_H4 & Re-test - test & 0.029 & 0.237 & 0.04 & 0.727 & 34 & 0.472 \\
\hline R Calc_H5 & Re-test - test & 0.038 & 0.33 & 0.055 & 0.686 & 34 & 0.498 \\
\hline L HW & Re-test - test & 1.344 & 8.06 & 1.361 & 0.987 & 34 & 0.331 \\
\hline L CBL & Re-test - test & 0.023 & 0.21 & 0.035 & 0.644 & 34 & 0.524 \\
\hline LCalc_HI & Re-test - test & 0.063 & 0.5 & 0.084 & 0.739 & 34 & 0.465 \\
\hline L Calc_H2 & Re-test - test & 0.053 & 0.29 & 0.049 & 1.08 & 34 & 0.288 \\
\hline L Calc_H3 & Re-test - test & 0.697 & 4.11 & 0.695 & 1.002 & 34 & 0.323 \\
\hline L Calc_H4 & Re-test - test & 0.716 & 3.86 & 0.651 & 1.098 & 34 & 0.28 \\
\hline L Calc H5 & Re-test - test & 0.643 & 3.5 & 0.592 & 1.086 & 34 & 0.285 \\
\hline
\end{tabular}

\section{Inkless collection footprints}

The differences between the digitally measured variables between trials were normally distributed, as assessed by visual inspection of a Normal Q-Q Plot of the right foot but showed some deviation from normality for some areas of the left foot in three values (Calc_H1, Calc_H2 and Calc_H5). However, as previous data sets had been used for analysis, the decision was taken to include these for further Paired-
Sample t-tests. Re-test scores in all variables tended to show larger scores than the original test for both feet. StdDev for two variables were unusual and showed a large discrepancy but matched two of the non normal Q-Q Plots -e.g. Left foot Calc_H1 and Calc_H2 (Table 4). These two StdDevs also are shown in the Paired Sample test results along with that of the Calc_H5 left foot, which showed high levels of StdDev (Table 5).

Table 4 Paired-Sample statistics for both feet digital measurement of the Inkless footprints

\begin{tabular}{|c|c|c|c|c|c|}
\hline \multicolumn{2}{|c|}{ R- Right Foot L - Left Foot } & \multirow{2}{*}{$\begin{array}{l}\text { Mean cm } \\
4.996\end{array}$} & \multirow{2}{*}{$\begin{array}{l}\mathbf{N} \\
35\end{array}$} & \multirow{2}{*}{$\begin{array}{l}\text { Std.Dev } \\
0.434\end{array}$} & \multirow{2}{*}{$\begin{array}{l}\text { Std. Error Mean } \\
0.073\end{array}$} \\
\hline R HW & Digital re-test & & & & \\
\hline & Digital test & 4.978 & 35 & 0.4 & 0.067 \\
\hline \multirow[t]{2}{*}{ R CBL } & Digital re-test & 9.065 & 35 & 0.658 & 0.111 \\
\hline & Digital test & 9.014 & 35 & 0.64 & 0.108 \\
\hline \multirow[t]{2}{*}{ R Calc_HI } & Digital re-test & 23.32 & 35 & 4.251 & 0.718 \\
\hline & Digital test & 23.28 & 35 & 4.252 & 0.718 \\
\hline \multirow[t]{2}{*}{ R Calc_H2 } & Digital re-test & 23 & 35 & 4.177 & 0.706 \\
\hline & Digital test & 22.98 & 35 & 4.158 & 0.702 \\
\hline \multirow[t]{2}{*}{ R Calc_H3 } & Digital re-test & 22.11 & 35 & 4.022 & 0.679 \\
\hline & Digital test & 22.08 & 35 & 4.007 & 0.677 \\
\hline \multirow[t]{2}{*}{ R Calc_H4 } & Digital re-test & 20.89 & 35 & 3.799 & 0.642 \\
\hline & Digital test & 20.89 & 35 & 3.785 & 0.639 \\
\hline \multirow[t]{2}{*}{ R Calc_H5 } & Digital re-test & 19.31 & 35 & 3.489 & 0.589 \\
\hline & Digital test & 19.28 & 35 & 3.477 & 0.587 \\
\hline \multirow[t]{2}{*}{ L HW } & Digital re-test & 5.076 & 35 & 0.469 & 0.079 \\
\hline & Digital test & 4.949 & 35 & 0.422 & $0.07 \mid$ \\
\hline \multirow[t]{2}{*}{ L CBL } & Digital re-test & 9.049 & 35 & 0.608 & 0.102 \\
\hline & Digital test & 9.026 & 35 & 0.511 & 0.086 \\
\hline \multirow[t]{2}{*}{ L Calc_HI } & Digital re-test & 24.062 & 35 & 1.324 & 0.223 \\
\hline & Digital test & 23.331 & 35 & 4.239 & 0.716 \\
\hline \multirow[t]{2}{*}{ L Calc_H2 } & Digital re-test & 23.62 & 35 & 1.194 & 0.201 \\
\hline & Digital test & 22.906 & 35 & 4.156 & 0.702 \\
\hline
\end{tabular}




\begin{tabular}{llllll}
\multicolumn{1}{l}{ Table continued } & \multicolumn{1}{l}{ R- Right Foot L - Left Foot } & Mean cm & N & Std.Dev & Std. Error Mean \\
\hline L Calc_H3 & Digital re-test & 22.065 & 35 & 4.033 & 0.68 I \\
& Digital test & 22.022 & 35 & 4.024 & 0.68 \\
L Calc_H4 & Digital re-test & 20.856 & 35 & 3.791 & 0.64 \\
& Digital test & 20.83 & 35 & 3.799 & 0.642 \\
L Calc_H5 & Digital re-test & 18.692 & 35 & 4.767 & 0.805 \\
& Digital test & $18.57 \mid$ & 35 & 4.714 & 0.796
\end{tabular}

Table 5 Paired-sample test for paired differences of digital footprint measurements of both feet using the inkless collection system

\begin{tabular}{llllllll}
\hline \multirow{2}{*}{ R-Right Foot; L-Left Foot } & \multicolumn{2}{c}{ Paired Differences mm } & \multirow{2}{*}{ t } & df & Sig. (2-Tailed) \\
\cline { 3 - 5 } & & Mean & Std.Dev & $\begin{array}{l}\text { Std. Error } \\
\text { Mean }\end{array}$ & & & \\
\hline R HW & Re-test - test & 0.018 & 0.163 & 0.027 & 0.673 & 34 & 0.505 \\
R CBL & Re-test - test & 0.05 I & 0.296 & 0.05 & 1.026 & 34 & 0.312 \\
R Calc_HI & Re-test - test & 0.031 & 0.299 & 0.05 & 0.622 & 34 & 0.538 \\
R Calc_H2 & Re-test - test & 0.015 & 0.269 & 0.045 & 0.339 & 34 & 0.737 \\
R Calc_H3 & Re-test - test & 0.031 & 0.297 & 0.05 & 0.62 & 34 & 0.54 \\
R Calc_H4 & Re-test - test & 0.002 & 0.283 & 0.047 & 0.048 & 34 & 0.962 \\
R Calc_H5 & Re-test - test & 0.026 & 0.286 & 0.048 & 0.555 & 34 & 0.583 \\
L HW & Re-test - test & 0.126 & 0.288 & 0.048 & 2.598 & 34 & 0.014 \\
L CBL_ & Re-test - test & 0.023 & 0.29 & 0.049 & 0.472 & 34 & 0.64 \\
L Calc_H1 & Re-test - test & 0.73 & 4.226 & 0.715 & 1.023 & 34 & 0.313 \\
L Calc_H2 & Re-test - test & 0.714 & 4.13 & 0.698 & 1.023 & 34 & 0.314 \\
L Calc_H3 & Re-test - test & 0.043 & 0.197 & 0.033 & 1.295 & 34 & 0.204 \\
L Calc_H4 & Re-test - test & 0.026 & 0.229 & 0.038 & 0.677 & 34 & 0.503 \\
L Calc_H5 & Re-test - test & 0.121 & 5.209 & 0.881 & 0.137 & 34 & 0.892 \\
\hline
\end{tabular}

The SEM's in the paired sample tests showed three high SEM's: one as high as .881 but apart from the three discrepancies the others showed SEM's from 0.050 to 0.027 . One pair showed a statistically significant result (left foot Heel width-t $(34)=2.598, \mathrm{p}=0.014$ ) (Table 5). Paired means showed a range from 0.731 to 0.002 with the right foot demonstrating lower mean values than those of the left foot in Calc H1, Calc_H2 and Calc_H5 which corresponded to the deviation from normality Q-Q Plots intimated above. Comparison between digital measurements of the two collection systems. Preliminary analyses, using scatter plots, showed the relationship of all the measurements to be linear with variables normally distributed, as assessed by ShapiroWilk test ( $>0.05)$, and there were no outliers. There was a strong positive correlation between the Podotrack measurements and those of the Inkless footprint as shown in Table 6.

Table 6 Pearson product-moment coefficient results for digital measurements comparing podotrack footprints with those of inkless shoeprint collection system

\begin{tabular}{llllllll}
\hline Left foot & & \multicolumn{7}{l}{ Right foot } \\
\hline Variable & Correlation & $\mathbf{r}$ & $\mathbf{r 2}$ & Variable & Correlation & $\mathbf{r}$ & $\mathbf{r 2}$ \\
\hline HeelWidth (HW) & Test Ink v Podo & $0.798^{* *}$ & 0.636 & HeelWidth (HW) & Test Ink v Podo & 0.006 & 0 \\
Cross Ball Line (CBL) & Test Ink v Podo & $0.934^{* *}$ & 0.872 & Cross Ball Line (CBL) & Test Ink v Podo & $0.95 I^{* *}$ & 0.904 \\
Calc_HI & Test Ink v Podo & -0.06 & -0.12 & Calc_HI & Test Ink v Podo & -0.61 & -1.22 \\
Calc_H2 & Test Ink v Podo & 0.142 & 0.02 & Calc_H2 & Test Ink v Podo & 0.177 & 0.03 I \\
Calc_H3 & Test Ink v Podo & $0.992^{* *}$ & 0.984 & Calc_H3 & Test Ink v Podo & $0.997^{* *}$ & 0.994 \\
Calc_H4 & Test Ink v Podo & $0.775^{* *}$ & 0.6 & Calc_H4 & Test Ink v Podo & $0.996^{* *}$ & 0.992 \\
Calc_H5 & Test Ink v Podo & $0.827^{* *}$ & 0.683 & Calc_H5 & Test Ink v Podo & $0.995^{* *}$ & 0.99 \\
HeelWidth (HW) & Re-Test Ink v Podo & 0.032 & 0.001 & HeelWidth (HW) & Re-Test Ink v Podo & $0.890^{* *}$ & 0.792 \\
Cross Ball Line (CBL) & Re-Test Ink v Podo & $0.909^{* *}$ & 0.826 & Cross Ball Line (CBL) & Re-Test Ink v Podo & $0.919^{* *}$ & 0.844 \\
\hline
\end{tabular}


Table continued

\begin{tabular}{|c|c|c|c|c|c|c|c|}
\hline \multicolumn{4}{|l|}{ Left foot } & \multicolumn{4}{|c|}{ Right foot } \\
\hline Variable & Correlation & $\mathbf{r}$ & r2 & Variable & Correlation & $r$ & r2 \\
\hline Calc_HI & Re-Test Ink v Podo & $0.898 * *$ & 0.806 & Calc_HI & Re-Test Ink v Podo & -0.022 & 0 \\
\hline Calc_H2 & Re-Test Ink v Podo & $0.943^{* *}$ & 0.889 & Calc_H2 & Re-Test Ink v Podo & 0.171 & 0.029 \\
\hline Calc_H3 & Re-Test Ink v Podo & 0.162 & 0.026 & Calc_H3 & Re-Test Ink v Podo & $0.997^{* *}$ & 0.994 \\
\hline Calc_H4 & Re-Test Ink v Podo & $0.995^{* *}$ & 0.99 & Calc_H4 & Re-Test Ink v Podo & $0.997 * *$ & 0.994 \\
\hline Calc_H5 & Re-Test Ink v Podo & $0.405^{*}$ & 0.164 & Calc_H5 & Re-Test Ink v Podo & $0.996 * *$ & 0.992 \\
\hline
\end{tabular}

** Correlation was significant at the 0.01 level ( 2 tailed)

*correlation was significant at the 0.05 level ( 2 tailed).

The strongest correlations occurred in the right and left feet of the test scores compared with those of the re-test scores. There were differences noted between feet and between tests. The left foot re-test scores showed lower correlations which in some cases were smaller in magnitude e.g. Heel width of the left foot re-test scores which showed $\mathrm{r}=0.032$. However the equivalent score for the right foot was 0.890 . There were differences in Pearson Product-moment correlations between feet as well as between test and re-test scores comparing the two collection systems. The highest correlations occurred in the Calcaneum to toe tip scores, especially of the lateral toes such as $3^{\text {rd }}$, $4^{\text {th }}$ and $5^{\text {th }}$ as evidenced in table 6 for Calc_H3, Calc_H4 and Calc_H5 of the right foot test and re-test scores. Moderately high scores of the left foot in test scores are also evidenced but not as high in re-test scores. The coefficient of determination $\left(\mathrm{r}^{2}\right)$ was high for the right foot in Cross Ball Line of the test scores, along with Calc H3, Calc H4 and Calc H5 but was poor for that of Heel width and especially of Calc H1 and Calc_H2 of test score values. With re-test scores the higher coefficient of determination occurred with the lateral three heel to toe measurements and cross ball width and heel width but again poor for the Calc H1 and Calc H2 measurements. In the left foot coefficient of determination were high for test scores of measurements apart from Calc_H1 and Calc_H2. Re-test results suggested high coefficients of determination for CBL, Calc H1, Calc_H2 and Calc H4 but not for heel width and Calc H3.

\section{Conclusion}

With the digital measurement there was a strong correlation between the two systems in both feet of the test scores compared with those of the re-test scores but there was greater fluctuations and differences in some variables e.g., in re-test right foot scores for heel width where correlation was $\mathrm{r}=.006$ and $\mathrm{Calc} \mathrm{H} 1$ and $\mathrm{Calc} \mathrm{H} 2$ being very low. It was not clear from the data as to why this occurred but some explanation may be postulated below. In the test and re-test scores Calc H3, Calc_H4 and Calc_H5 of the right foot especially demonstrated the highest correlation. This might suggest this side of the foot (lateral side) has greater stability than that of the inner side (medial side), which has to adapt to the ground or surface more and to allow leverage and propulsion through the great and second toes. Why the difference between the two collection methods and feet is unclear but the following is offered as some postulated theories as to differences in the results overall. The Podotrack produced much darker images than the inkless system, which might account for some variation between the two systems in terms of where measurements were taken and might account for the differences between test and re-test results. The Podotracks have a relatively indistinct edge or outer border throughout the footprint, which made it difficult even in Photoshop $\subset$ to determine the exact edge where the measurement should occur.
This could result in errors of measurement, which might be two fold-the commencement point and the end point might vary between tests and re-test results, in other words there are inconsistencies between the start and end points defined for the measurement. Participants did not have as much difficulty 'targeting' the Podotrack as there was only one area to step onto compared with the two for the Inkless system, which might have reduced error in measurement because the gait pattern might have been anticipated to be uninterrupted using the Podotrack compared with that of the Inkless collection system. There is controversy within the literature about the concept of targeting. Whittle ${ }^{40}$ for example suggested visual guidance, or 'targeting' the inkless sheet may lead to an artificial gait pattern, as the subjects 'aim' for the platform' (page 149). However, other studies investigating the effects of targeting on ground reaction force variability and the temporo-spatial parameters of gait have shown no statistical differences between non-visually and visually guided steps [41-43]. Therefore this was not considered an issue within the current study and participants were allowed to target the inkpad, the paper or the Podotracks.

Due to the various sizes of feet, along with the issues of paper and collection system dimensions, targeting of the pad and the paper/ Podotrack occurred and although not encouraged helped/assisted in gaining appropriate footprints ensuring all areas were captured. The current study used a $4^{\text {th }}$ step protocol that appeared to reduce the amount of wastage and also targeting compared with the 6th step protocol used in a pilot study. Participants appeared to find it more difficult to "land 'on the inkpad and then the paper when they were placed further down the walkway and inappropriate or incomplete footprints were attained. The footprints were partial as the participants had only partially landed on the inkpad, or they found it more difficult to land on the paper after 6 steps compared with 4 and only partial prints were obtained with the toes missing or the heel area missing. These were more common issues than losing part of the foot to one side. Overall the inkless system allowed a clearer, better-defined image with better landmarks, dermatoglyphics (not within scope of practice of forensic podiatrists but usable by the Criminal Justice System (CJS) to assist identification) and fewer artefacts than the Podotrack system of collection.

However, it was unknown if there are any allergies likely to be caused by the unknown chemical in the inkless system. As the system was not designed or sold for footprint collection but for shoe collection by a technician it was unknown whether any tests or studies have been conducted to ensure the safety of the system against skin allergies. The results suggested differences between feet, which justified the studies design in obtaining both feet. However, as the results were reported as two separate entities (left and right feet independently and calculated independently) the statistical results should not be compromised. In 
forensic practice comparison is between the unknown and the known therefore it is likely that only one side of the body is being compared. But as the CJS requires practitioners to be able to determine SEM's then which side is being investigated requires the correct SEM to be applied. The fact the results showed that there were differences, caution is needed with assuming there will be no differences between two sets of data, between two different collection techniques or measurement processes and should not be seen as a problem within the forensic community. However this study clearly showed that correlation between variables within the foot /feet was inconsistent and therefore supports the notion that both feet should be used. The author would recommend using the Inkless collection system as preference to the Podotracks but one collection system is as good or bad as the other in terms of footprint collection. However, in forensic practice it is more likely that another system for footprint collection would be required which would enable multiple steps to be collected at the same time on the same paper based system, either as a roll of paper or a large area onto which numerous footprints could be collected easily.

\section{Acknowledgments}

None.

\section{Conflicts of interest}

The author declares that there are no conflicts of interest.

\section{Reference}

1. Kanchan T, Krishan K, ShyamSundar S, et al. Analysis of footprint and its parts for stature estimation in Indian population. Foot. 2012;22(3):175-180.

2. Kanchan T, Krishan K, Sharma A, et al. A study of correlation of hand and foot dimensions for personal identification in mass disasters. Forensic Sci Int. 2010;199(1-3):112.

3. KrishanK. Estimation of stature from footprint and foot outline dimensions in Gujjars of north India. Forensic Sci Int. 2008;175(2-3):93-101.

4. Jasuja OP, Harbhajan S, Anupama K. Estimation of stature from stride length while walking fast. Forensic Sci Int. 1997;86(3):181-186.

5. Jasuja OP, Manjula. Estimation of stature from footstep length. Forensic Sci Int. 1993;61(1):1-5.

6. Jasuja OP, Singh J, Jain M. Estimation of stature from foot and shoe measurements by multiplication factors: a revised attempt. Forensic $S \mathrm{Ci}$ Int. 1991;50(2):203-215.

7. Aurichio TR, Rebelatto JR, de Castro AP. The relationship between the body mass index (BMI) and foot posture in elderly people. Arch Gerontol Geriatr. 2011;52(2):e89-e92.

8. Fawzy IA, Kamal NN. Stature and body weight estimation various footprint measurements among Egyptian population. J Forensic Sci. 2010;55(4):884-888.

9. Oladipo G, Bob-Manuel I, Ezenatein G. Quantitative comparison of foot anthropometry under different weight bearing conditions amongst Nigerians. The Internet Journal of Biological Anthropology. 2009;3(1).

10. Atamturk D, Duyar I. Age-related factors in the relationship between foot measurements and living stature and body weight. J Forensic Sci. 2008;53(6):1296-1300.

11. Grivas TB, Mihas C, Arapaki A, et al. Correlation of foot length with height and weight in school age children. $J$ Forensic Leg Med. 2008;15(2):89-95.

12. Krishan K. Determination of stature from foot and its segments in a north Indian population. Am J Forensic Med Pathol. 2008;29(4):297-303.
13. Bavdekar SB, Sathe S, Jani P. Prediction of Weight of Indian Children Aged upto Two Years Based on Foot-length: Implications for Emergency Areas. Indian Pediatr. 2006;43(2):125-130

14. Sen J, Kanchan T, Ghosh S. Sex estimation from foot dimensions in an indigenous Indian population. J Forensic Sci. 2011;56(1):S148-S153.

15. Atamturk D. Estimation of sex from dimensions of foot, footprints and shoe. Anthropol Anz. 2010;68(1):21-29.

16. Zeybeck G, Ergur I, Demiroglu Z. Stature and gender estimation using foot measurements. Forensic Sci Int. 2008;181(1-3):54.

17. Ozden $H$, Balci $Y$, Demirüstü $C$, et al. Stature and sex estimate using foot and shoe dimensions. Forensic Sci Int. 2005;147(2-3):181-184.

18. Woods TS. Expert opinion evidence invoking "novel science": A look at some Canadian case law. The Expert and Dispute Resolver no Summer. 2009;9-14 p.

19. Loomis SB. The Daubert test of reliability: Fighting "junk science" in the courtrooms. SketicReport. 2002;1-11 p.

20. Naples VL, Miller JS. Making tracks: The forensic analysis of footprints and footwear impressions. Anat Rec B New Anat. 2004;279(1): 9-15.

21. Fraser J, Williams R. Handbook of forensic science. Willan Publishing, USA. $2009 ; 1-70$ p.

22. Morrison GS. Forensic voice comparison and the paradigm shift. $S c i$ Justice. 2009;49(4):298-308.

23. National Research Council. Strengthening forensic science in the United States - A path forward. National Academy of Science, Washington, USA. 2009.

24. Skills for Justice. National Occupational Standards for Forensic Science. UK. 2011.

25. Andrew Rennison. Forensic Science Regulator. Codes of practice and conduct (Version 2.0). UK. 2011;2-56 p

26. Vernon W, McCourt FJ. Forensic podiatry - a review and definition. British Journal of Podiatry. 1999;2(2):45-48.

27. Dror I. The ambition to be scientific: Human expert performance and objectivity. Sci Justice. 2013;53(2):81-82.

28. Vernon W. Formal recognition of forensic podiatry by the International Association for Identification (IAI). Podiatry Now. 2007;10(11):42-42.

29. Vernon W, Brodie B, DiMaggio J, et al. Forensic podiatry: Role and scope of practice (in the context of forensic human identification). International Association for Identification, USA. 2009;1-17.

30. The Law Commission. Expert evidence in criminal proceedings in England and Wales. The Stationary Office, The House of Commons, London, USA. 2011.

31. DiMaggio JA. The emerging science of forensic podiatry: This new specialty is open to DPM's with investigative minds. Podiatry Management. 2005;24(6):147.

32. Juster HR, Heimberg RG, Engelberg B. Self-selection and sample selection in a treatment study of social phobia. Behav Res Ther. 1995;33(3):321-324.

33. Meyers-Rice B, Sugars L, McPoil T, et al. Comparison of three methods for obtaining plantar pressures in non-pathologic subjects. $J$ Am Podiatr Med Assoc. 1994;84(10):499-504.

34. Reel S. Development and evaluation of a valid and reliable footprint measurement approach in forensic identification. $\mathrm{PhD}$ Thesis, University of Leeds, York St John University. 2012.

35. Reel S, Rouse S, Vernon W, et al. Reliability of a two-dimensional footprint measurement approach. Sci Justice. 2010;50(3):113-118. 
36. Gunn N. Old and new methods of evaluating footprint impressions by a forensic podiatrist. British Journal of Podiatric Medicine and Surgery. 1991;3(3):8-11

37. Scientific Working Group Imaging Technology (SWGIT). Section 1, Overview of SWGIT and the Use of Imaging Technology in the Criminal Justice System. 2007b.

38. Scientific Working Group Imaging Technology (SWGIT). Section 12 Best Practices for Forensic Image Analysis. 2007a.

39. Cohen J. Statistical power analysis for the behavioural sciences, 2nd ed Lawrence Erlbaum Associates publishers, USA. 1988.
40. Whittle M. Gait analysis: an introduction, 4th ed. ButterworthHeinemann publishers, UK. 2007.

41. Wearing SC, Urry SR, Smeathers JE. The effect of visual targeting on ground reaction force and the temporo-spatial parameters of gait. Clin Biomech (Bristol, Avon). 2000;15(8):583-591.

42. Grabiner MD, Feuerbach JW, Lundin TM, et al. Visual guidance to force plates does not influence ground reaction force variability. J Biomech. 1995;28(9):1115-1117.

43. Sanderson DJ, Franks IM, Elliot D. The effects of targeting on the ground reaction forces during level walking. Human Movement Science. 1993;12(3):327-337. 